\title{
The Four Cs of Psychological Wellbeing: Lessons from Three Decades of Computer-based Environmental Enrichment
}

\begin{abstract}
David A.Washburn ${ }^{1 *}$
${ }^{1}$ Georgia State University

*Corresponding author (Email: dwashburn@gsu.edu)

Citation - Washburn, D. A. (2015). The four Cs of psychological wellbeing: Lessons from three decades of computerbased environmental enrichment. Animal Behavior and Cognition, 2(3), 218-232. doi: 10.12966/abc.08.02.2015

Abstract - Three decades ago, the Animal Welfare Act was amended to require researchers to provide environments that promoted the psychological, as well as the physical, wellbeing of nonhuman primates maintained for research purposes. We developed a computer-task paradigm with the goal of promoting and assessing the psychological wellbeing of rhesus monkeys. Some lessons learned in the three decades of using this enrichment strategy are discussed in this review. These findings underscore the importance of comfort, companionship, challenge and control in the psychological wellbeing of nonhuman primates, and the utility of game-like computerized tasks for addressing these dimensions of psychological fitness.
\end{abstract}

Keywords - Environmental enrichment, Psychological wellbeing, Rumbaughx, Comfort, Challenge, Companionship, Control

The conquest of space is also the conquest of physical frailty. When President John F. Kennedy committed to put an American on the moon by the end of the 1960s, he launched a national initiative that focused science and technology on the exploration of space. What is equally true but less obvious is the extensive program of research (domestic and abroad) on human physiology and function that was also mobilized by this ambitious aim. The challenges faced by the National Aeronautics and Space Administration (NASA) were as much about musculoskeletal morphology, circulatory systems, neurovestibular function, and circadian patterns of performance as they were about rockets, guidance systems, communications and the like. A quarter-century after President Kennedy's 1961 speech, NASA had accomplished a long and impressive record of success in sending humans and other animals into space — indeed, onto the surface of the moon - and safely home; however, many questions remained unanswered regarding how the human body reacts to exposure to microgravity and the other conditions of (particularly extended) spaceflight. Even today, it seems clear that the most intractable obstacles and puzzles regarding life in space are less about space and more about life.

It is in this context of understanding how humans adapt, or fail to adapt, physically and psychologically to spaceflight that an international research program was initiated in 1983. A partnership of the American, European, and Russian space agencies, the project was designed to study comprehensively and in great detail how physiological systems change over time during 14-day spaceflight. For this work, a nonhuman primate model was selected for study, so that microgravity-related changes in skeletal, muscular, cardiopulmonary, digestive and microbiological, immunological, neurovestibular, and regulatory systems might be identified. Named for this primate model, the so-called Rhesus Project planned a 14-day space shuttle mission with macaques living as payload, continuously monitored and behaving with minimal interaction with the human crew. An impressive international and interdisciplinary team of scientists was 
assembled, including many of the most respected Russian, French, and American scholars interested in gravitational and space biology.

The 1985 amendment to the Animal Welfare Act created a new challenge for this team. Among other changes, the amendment required researchers to "...provide a physical environment adequate to promote the psychological wellbeing of primates" [United States Code, 7, Section 2143, (a)(2)(B) Supp. IV 1986]. In addition to all of the plans and safeguards being implemented to ensure the physical health and wellbeing of the rhesus monkeys, it would be necessary to provide for the animals' psychological wellbeing before, during, and after the spaceflight. Although environmental enrichment and psychological wellbeing of nonhuman animals were certainly not new research domains, it is accurate to say that the AWA amendment sparked a flurry of studies, speculations, and debates across research community about the best ways to ensure and to document compliance. The Rhesus Project was by no means alone in struggling with the best ways to provide environmental enrichment that would promote the psychological wellbeing of the captive macaques maintained for that research.

Rhesus Project leaders solicited the expertise on this issue of noted primate behavioral researcher Professor Duane M. Rumbaugh of Georgia State University. With decades of innovative behavioral studies of primate learning, language, and other cognitive competencies, Rumbaugh's experience in studying captive apes and monkeys made him highly qualified to talk about their psychology. In a meeting with Rhesus Project coordinators, Rumbaugh recounted many of the interventions and activities that appeared to be effective in promoting the psychological fitness of the small resident colony of apes at Georgia State University's Language Research Center (LRC). In particular, he noted the apes' apparent enjoyment of a relatively new research paradigm that was being used at the LRC. For several prior years, questions about chimpanzee learning, memory, symbol use, numerical cognition, and so forth had been translated into simple, game-like computer tasks, making use of the first wave of "personal computers" commercially available and suitable for relatively simple versions of the arcade video games that were at the time generating billions of dollars at 25 cents per play. [As a personal aside, my own first assignment as a graduate assistant in 1984 was programming such game-like tasks for the apes, and perhaps my greatest contribution in those early years was to transition the LRC from machine-coded systems to new Commodore 64 computers and the BASIC-language platform for joystick-based game-like computerized tasks.] Significantly - and anticipating a theme for discussion below - the apes had indicated their affinity for computer-based testing by their own behavior. That is, these animals chose to work on joystick-based tasks, even though they did not need to perform the game-like tests in order to receive food or to have something to do. The apes opted for computer-task activity over other potential activities that were available to them. Rumbaugh reasoned that rhesus monkeys too might enjoy performing these tasks, although there were substantial reasons (see Rumbaugh et al., 1989) to believe that rhesus monkeys might not understand the discontiguous cause-effect relations necessary to respond to control a computer-graphic cursor by manipulating a joystick. (Thus, in parallel with joystick training, work was also initiated with an additional rhesus monkey using a more costly touchscreen option.) Armed with IBM's new DOS-based entries into the growing personal-computer market, Professor Rumbaugh and his research team developed a curriculum of computerized game-like tasks that increased in difficulty both across trials and across tasks. These tasks were presented to two macaques obtained from NASA, Abel and Baker, who became the initial explorers for their species of this new digital world.

Almost 30 years, many dozens of animals, and hundreds of publications later, it is clear that many nonhuman primate species - chimpanzees and rhesus monkeys, but also New World monkeys, other Old World primates, other apes, and even prosimians - can readily learn to manipulate a joystick or use a touchscreen so as to respond to computer-graphic stimuli in accordance with the rules of a host of gamelike tasks (e.g., Andrews, 1993; Beran \& Minahan, 2000; Brannon, Andrews, \& Rosenblum, 2004; Evans, Beran, Chan, Klein, \& Menzel, 2008; Fagot, Gullstrand, Kemp, Defilles, \& Mekaouche, 2014; Fagot \& Paleressompouille, 2009; Fragaszy et al., 2009; Hopkins, Washburn, \& Hyatt, 1996; Inoue \& Matsuzawa, 2009; Jones et al., 2014; Leighty \& Fragaszy, 2003; Menzel \& Menzel, 2007; Tanaka \& Uchikoshi, 2010; Vonk et al., 2014). The paradigm has been used successfully to study a wide range of cognitive phenomena, including learning, memory, attention, perception, categorization, numerical cognition, problem solving, 
reasoning, decision making, metacognition, social cognition, and language (e.g., Fagot \& Bonté, 2010; Spinelli et al., 2004; Washburn \& Rumbaugh, 1992a). The focus for the present paper, however, is that original topic that inspired Professor Rumbaugh and his collaborators to give rhesus monkeys dedicated new computers: psychological wellbeing, and the question of whether computer-based tasks such as these serve as environmental enrichment consistent with the demands of the AWA amendment. Evidence for the enrichment value of the computer-test paradigm, also called the video-task paradigm, the computerized or psychomotor test system, or the "Rumbaughx" (pronounced "RUM-box"; Washburn, Beran, Evans, Hoffman, \& Flemming, 2013) - to put the apparatus in context with other classic comparative instruments like the puzzle box, shuttle box, and Skinner box - has been reported elsewhere (e.g., Washburn, 2003; Washburn, Harper, \& Rumbaugh, 1994; Washburn \& Rumbaugh, 1992b; Washburn, Rumbaugh, \& Richardson, 1992; for similar reports from other settings, see also Clay, Perdue, Gaalema, Dolins, \& Bloomsmith, 2011; Fagot et al., 2014; Mallavarapu, Bloomsmith, Kuhar, \& Maple, 2013; Perdue, Clay, Gaalema, Maple, \& Stoinski, 2012; Platt \& Novak, 1997; Torou, Kuhar, Adcock, Bloomsmith, \& Maple, 2004; Yamanashi \& Hayashi, 2011). The goal for the present paper is to overview this evidence, organized as a nonexhaustive list of lessons from three decades of using the strategy for environmental enrichment. The focus will be the methods and findings from the Language Research Center, but it should be acknowledged that investigators at many other laboratories have used the paradigm successfully, in some instances developing innovative variations (e.g., touchscreens versions, use for testing social groups) that are remarkable in their own right (e.g., Fagot \& Bonté, 2010; Gazes, Brown, Basile, \& Hampton, 2013). Similarly, the exclusive focus here on primates belies the high probability that the same issues of psychological wellbeing, and the same benefits from computer-based interventions, apply to other types of animals.

\section{Method}

The findings and the lessons reported here emerge from a methodology that has been described previously (e.g., Rumbaugh et al., 1987; Washburn et al., 2012). Although there have been some variations across the years, some elements of this methodology are largely consistent. At the LRC, rhesus monkeys (Macaca mulatta), ranging from about 1 year to over 30 years in age, have been trained to respond to computer-graphic stimuli by manipulating joysticks that controlled cursors on dedicated test-system screens (i.e., one computer, monitor, joystick, dispenser, external speaker per monkey; see Figure 1). Because of the NASA project that funded and motivated the work in its first decade, the macaques at the LRC have almost exclusively been males, typically singly housed in column-style cages with continuous visual and auditory access to one another. Compatible pairs of monkeys have been moved into an indoor/outdoor housing area regularly (usually one day/week). All animals had continuous access to the joystick and a suite of tasks whenever they were in their home cages, including during the overnight period. In some periods, the animals were presented with menus of icons representing the tasks that were available to them (Washburn, Hopkins, \& Rumbaugh, 1991), whereas at other times the different tasks cycled through across time. In either case, the animals typically had opportunities to work on multiple tasks each day, so that some data were obtained daily for all the studies in which a monkey was participating. In all instances, the monkeys worked on their dedicated test systems when they wanted and rested or engaged in other activities (e.g., sleeping, playing with other manipulanda such as Kong toys or puzzles) when they chose. That is, no monkey was deprived of food or fluid or reduced in body weight for purposes of testing. During the work day, food available was generally restricted to the fruit-flavored chow pellets dispensed when computertask trials were successfully completed, but supplemental chow, fruit and vegetables were provided dailywhether or not the animals worked - to maintain each monkey's weight. Incorrect responses typically produced auditory feedback, frequently accompanied by a time-out period. No other punitive method was used to promote productivity, accuracy, or rapid responding. Water was always available to these resident animals. 


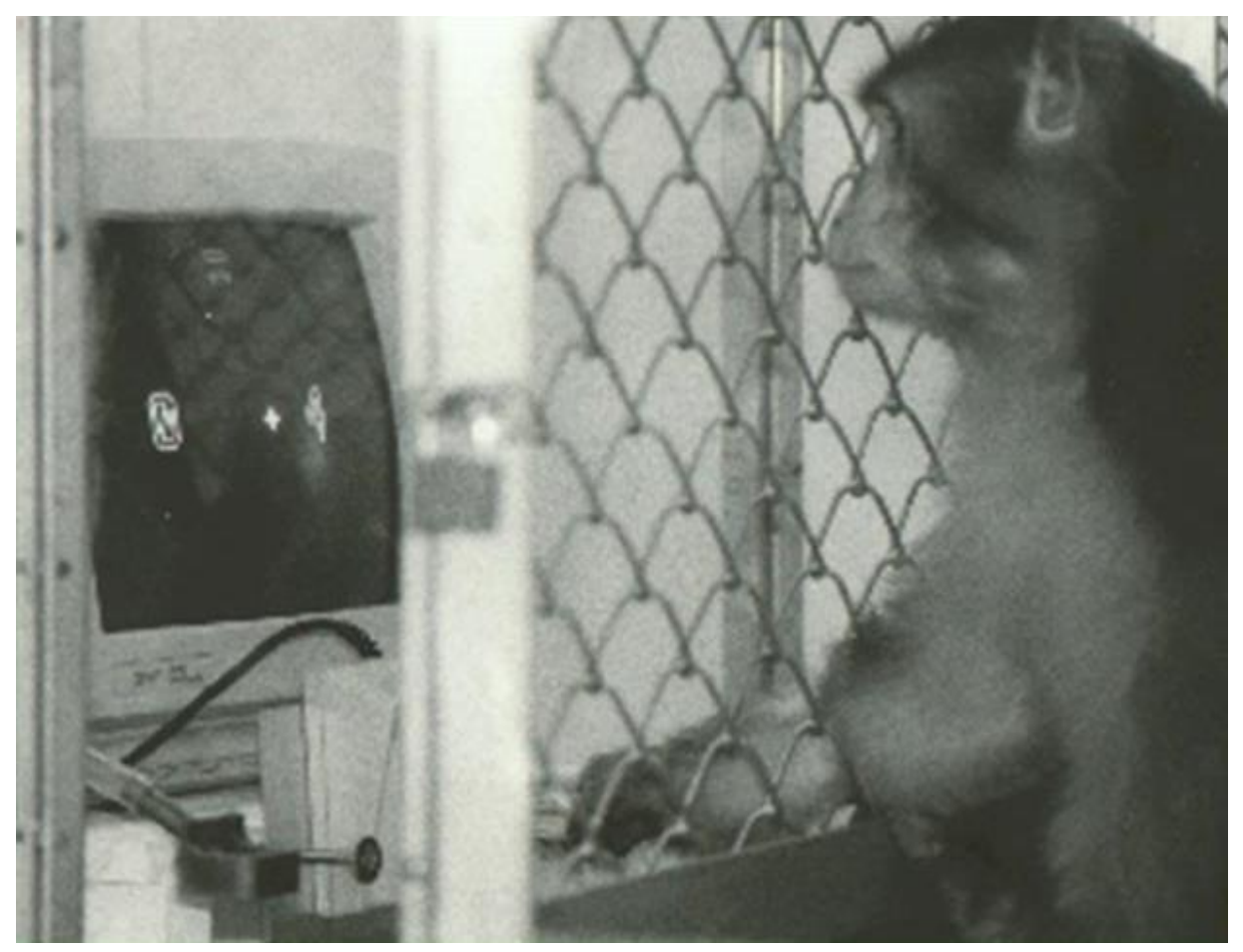

Figure 1. A monkey reaches through the mesh of his cage to manipulate a joystick in a typical Rumbaughx setup.

The monkeys tested in this fashion 7 days/week, 365 days/year (except on days that they were pairhoused in the indoor/outdoor social cage). New tasks were introduced regularly, but the monkeys were also able to engage older, preferred tasks. Because Georgia State University and the LRC are committed to support and test these animals for their natural lifetimes (i.e., from whatever age they were when acquired for the project, until death by natural causes or euthanasia for veterinary and humane reasons in instances of terminal illness), they provide unique and important opportunities to study how behavior changes over time, as when new cognitive competencies emerge on the scaffolding of abilities that developed through earlier experience and training. That is, just as cognitive sophistication increases across child development, not merely as a function of time and maturation but also as a function of accumulated experience, so we may see new, previously undocumented competencies in rhesus monkeys that bring ever-increasing repertoires of prior learning to bear on new cognitive challenges.

\section{Lesson 1: It is Easier to Measure the Absence of Wellbeing than its Presence}

In their seminal paper on the challenges faced by primate researchers in response to the 1985 AWA amendment, Novak and Suomi (1998) noted that it would be difficult to reach consensus on the environment sufficient to promote the psychological wellbeing of our own species, much less a nonverbal, nonhuman primate species. Nevertheless, the federal mandate combined with the broadly shared ethical motivation to provide the animals with the best care possible made environmental enrichment an important topic in primate research. Novak and Suomi proposed an integrative approach to the assessment of psychological wellbeing, where a nonhuman primate is considered psychologically well if two of four criteria are satisfied: (a) The primate is in good physical health; (b) it exhibits a substantial range of the species' behavioral repertoire and, if individually housed, does not display high levels of bizarre, stereotyped, or disorganized patterns of behavior; (c) it is not in a chronic state of distress; and (d) it is able to respond effectively to environmental challenges. Note that for at least three of these criteria, the measurement of psychological wellbeing is at least as much about the absence of evidence of psychological impairment as it is the positive evidence of wellness. That is, the psychological wellbeing of nonhuman primates is marked by the absence 
of symptoms of physical illness, distress, stereotypies, bizarre or maladaptive behavior, and so forth. Only the range of species-typical behavior is entirely positive. More frequently, psychological wellbeing is inferred by the absence of indicators of psychological compromise, just as physical wellbeing is generally assumed-in nonhuman animals as in our own species-in the absence of symptoms of malaise, discomfort, and disease.

Similarly, Bayne (1989) summarized recommendations for the assessment of psychological wellbeing provided by veterinarians and primate researchers. Both lists tended to emphasize evidence of psychology distress rather than wellbeing per se. Veterinarians noted the meaningfulness of stereotypies, overgrooming, self-injurious behavior or other forms of aggression, distress vocalizations, decline in appetite or activity, over-reactivity to stimuli, and alterations in physical appearance. Researchers similarly described physiological (e.g., cortisol levels), physical (quality of hair coat), behavioral (attentiveness, aggression), and performance-based indicators of psychological wellbeing, where again the environment is inferred to be adequate to promote the psychological wellbeing of nonhuman primates to the degree that signs of stress and distress are absent.

At the LRC and in parallel training of more than 100 rhesus monkeys for NASA, both at Ames Research Center in California and the Institute for Biomedical Problems in Moscow, Russia, many different strategies were employed to rate the psychological wellbeing of the captive monkeys maintained for this research. For example, caretakers observed and rated each monkey on a scale ranging from "much more inactive or depressed than normal" to "much more active or agitated than normal," with a central value representing each animal's typical level of activity and interactivity. Not surprising however, this strategy resulted in many weeks or even months of ratings around " $3=$ normal" punctuated by the occasional brief " 1 = very inactive" or " 5 = very agitated" rating corresponding to a period of illness or stress (e.g., a vet visit or construction work), respectively. Analyses of the correlates and predictors of variations in psychological wellbeing scored in this way were generally uninformative, because there was little variability to be explained by environmental conditions, performance levels, and so forth. More detailed rubrics for scoring animal behavior were also tried. These took longer to produce and yielded a finer-grain analysis of each monkey's behavior (e.g., all reasons for inactivity or signs of aggression are not equally maladaptive, of course), but again the day-to-day variability in these assessments was small, presumably because the variability in psychological wellbeing of animals maintained in relatively comfortable and enriched environments also varied little across days. Critically, no drift in wellbeing scores across time were observed with this more detailed behavioral rubric; that is, scoring behavior scaled to each animal's "normal" level runs the risk of gradual changes that would be obvious and worrisome if assessed at two discrete time points, but that might be concealed by a sliding sense of what is typical for any individual animal that becomes progressively more agitated, stressed, distressed, depressed and so forth. Detailed behavioral codes did not reveal any such progressive change, but rather confirmed stable levels of wellbeing, which stood in sharp and empirically obvious contrast to rare incidents of illness, injury, conflict, and stress.

As a consequence, I adopted and have previously described (e.g., Washburn, 2003) a "wellbeing index" measured as the percentage of observed time in which an animal does not appear to be experiencing psychological compromise. Rather than a rubric for scoring psychological wellbeing per se, this is a measure of the absence of psychological distress, where the proportion of time spent in overgrooming, selfinjury, stereotypy, distress vocalization, and similar undesirable behaviors represents the inverse of wellbeing. This is certainly not a perfect metric, as it ignores potential variability within psychological wellbeing, such as changes in mood or stress that are below the threshold for overt behavioral manifestations of psychological compromise. For example, Gulledge, Rumbaugh, Fernandez-Carriba, and Washburn (2015) recently reported data in which untrained observers coded the countenance of still photographs of rhesus monkey faces. Photographs of animals that had access to indoor/outdoor caging were scored as happier than animals with only indoor caging, even though the lighting, background, and other conditions that might distinguish between these two groups was otherwise controlled. That is, untrained observers scored a group of monkeys with a relatively more-enriched environment as happier looking than the other group with relatively less-enriched caging - although neither group was showing signs of 
psychological distress that would have reduced their score on the wellbeing index discussed here and previously.

Nevertheless, for the present report the wellbeing index, or proportion of time an animal spends in psychology healthy behaviors, was employed. To compute the wellbeing index, a schedule of pseudorandom times was generated for each monkey, and video recordings were taken at each scheduled time. This permitted unobtrusive observation of each monkey's behavior at various times throughout each day, without modification by the animal's reaction to or interaction with the human observer. Each video was independently coded by two or more observers, so as to produce an activity budget for each animal, indicating the proportion of time spent sleeping, pacing, rocking, eating, manipulating toys or other cage items, engaging in task-related behavior, and so forth. Distress vocalizations and displays were also counted.

In the baseline condition (singly housed males with no computer-task available, but with other common primate toys in the cage and conspecifics in neighboring cages in the same test room), about half of the observations included sleeping or resting. Self-directed behavior, ranging from grooming to selfinjury, was observed on $14 \%$ of the samples. Rocking, pacing or other stereotypy was observed on $20 \%$ of the samples. Engagement of a manipulandum that could be baited with food (e.g., Kong toys, foraging cloths, other puzzles) accounted for $13 \%$ of the observations, primarily at those times shortly after the item was made available and until the food had been obtained.

It is not surprising that this behavioral ethogram changed when compatible conspecifics were paired together in a cage, or when the computer-task apparatus was made available. These conditions provided opportunities for behaviors (e.g., grooming others, task engagement) not possible in the baseline condition. What is more important is what behaviors would be replaced by the new options. Would the changes from pair housing improve psychological wellbeing, relative to single-animal housing? Would joystick access compete with the use of other enrichment devices, or perhaps alter sleeping habits - as seems to happen with so many human adolescents? Or would computer-task engagement occupy time that was previously associated with relatively undesirable behaviors?

\section{Lesson 2: Computer-task Testing is an Effective Environmental Enrichment for Promoting Psychological Wellbeing}

Figure 2 shows the answer to these questions: The data reported by Washburn and Rumbaugh (1992a) have been regraphed as difference scores relative to the baseline data discussed above. Numbers above the midline indicate a decrease in observed samples with relatively undesirable or maladaptive behavior (e.g., self-injury, stereotypy, distress vocalizations), and thus a higher wellbeing index. For comparison purposes, values are also presented for two social isolation conditions in which singly housed monkeys were maintained for short periods in a room without other conspecifics, with and without access to the computerized test system. Two findings are immediately apparent from these data: First, the social manipulation had pronounced effects on psychological wellbeing, as fewer indicators of psychological distress were observed in the pair-housed versus the singly housed versus the social isolated condition (irrespective of access to the computerized test system). Relative to the baseline condition, the wellbeing index value dropped more than $30 \%$ during periods of social isolation, but improved by about $20 \%$ when compatible animals were pair-housed. 


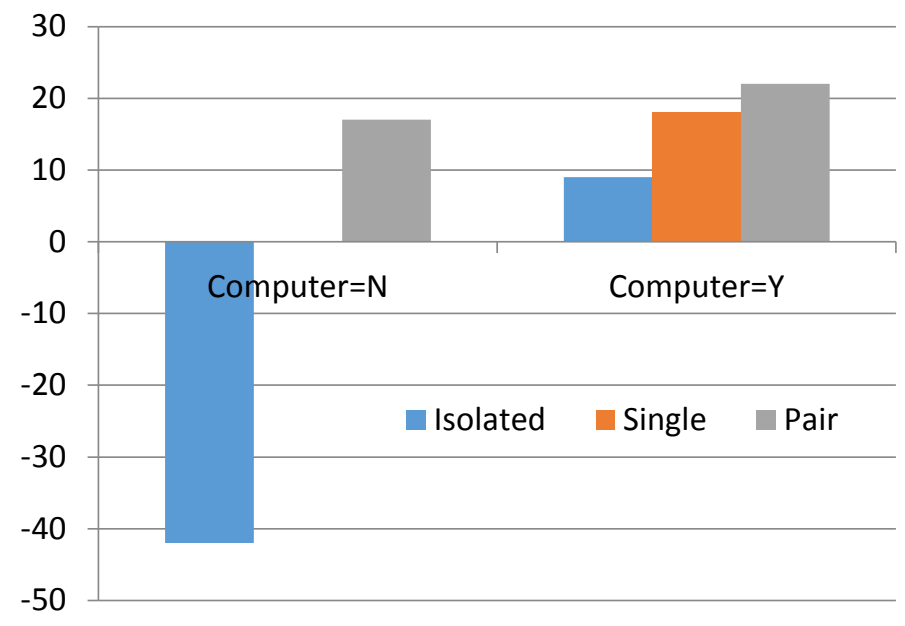

Figure 2. Change in Wellbeing Index as a function of manipulations of social housing and computer-task availability

The second finding that is obvious from this figure is that access to the computerized test system was also potent in improving psychological wellbeing, even moderating the pronounced effects of social isolation. Overall, wellbeing index values increased by about $15 \%$, relative to baseline, across socialhousing conditions. With access to the computerized test system, the monkeys continued to direct attention toward other manipulanda (toys and puzzles) in the cage, at least when those items were baited with treats. The change in each monkey's activity budget was a reduction in the number of observations associated with behaviors like rocking, pacing, huddling, distress vocalizing, overgrooming, and aggression. I have previously interpreted these data as indicating that the computerized test system is even more effective than social-housing for supporting a monkey's psychological wellbeing, as indicated by the insulating effect that computer-task access appears to produce even during periods of social isolation. When the wellbeing-index data are rescaled as percent-difference scores as in Figure 2, it seems clear that a more comprehensive conclusion is that social isolation does substantial harm to psychological wellbeing (although this effect can be minimized by access to a computerized test system), and that computer-based enrichment and pairhousing of compatible animals produce additive effects that are greater than the wellbeing values of other environmental enrichment manipulanda.

Of course, it is clear why it might be true that the computer-based test system improves psychological wellbeing more than other effective manipulanda, such as toys and puzzles. As described above, rhesus monkeys are highly engaged by Kong toys, foraging cloths, and other puzzles that are baited with treats, at least until the food has been exhausted. Once the peanut butter has been licked off, the pellet crumbles have been groomed off, the seeds and nuts have been foraged out, and the puzzle has been solved, there is typically a dramatic decrease in interest - until the manipulandum is again re-baited. The computerized test system is essentially a foraging manipulandum that automatically replenishes itself each trial. Computer-based tasks provide a wide range of problems to be solved, but the rewards of success do not stop so long as the pellet dispenser (or juice dispenser, universal feeder, video/auditory reward device) remains loaded. A Kong toy that somehow automatically refilled with treats or a foraging field that continuously replenished with edibles - as indeed happens in nature - would likely be similarly effective in promoting psychological wellbeing by generating activity-directed behavior that replaces signs of boredom or distress (e.g., rocking, pacing, huddling, overgrooming) in the animals' behavioral ethograms. 


\section{Lesson 3: There is Value in Natural Enrichment}

In recent discussions about the continued testing of chimpanzees in behavioral and biomedical studies in the United States, it has been argued that the laboratory environment should be as natural as possible for nonhuman primates. Natural climbing structures and substrates, browse and foraging materials, and activities certainly look more enriching, and may empirically be more enriching, compared to the cement floors and metal bars that are brought to mind (accurately, for the most part) when one thinks of a laboratory. There are complexities to the balance between a completely naturalistic environment on the one hand and an easily sanitized and reasonably affordable environment on the other. To the best of my knowledge, no nonhuman primate has been given the free choice of living in an environment that is as similar to the wild as is safely possible, versus living in what we would think of as the humans' natural environment, complete with comfortable chairs and beds, entertainment options, and access to food. There have certainly been demonstrations that the physical and psychological wellbeing of animals improve in naturalistic environments compared to traditional, sterile laboratory or zoo caging (e.g., Clarke, Juno, \& Maple, 1982; Ogden, Lindburg, \& Maple, 1993). However, this is different than comparing a natural or naturalistic environment to a comfortable (human) home-like environment. Sue Savage-Rumbaugh addressed this issue with respect to the apes she raised and tested in language-research projects at the LRC, and speculated that given the choice the animals would choose the comforts of the human-like environment (National Geographic Explorer video, Pygmy Chimps: Star Students, 1986). In any case, the importance of naturalism remains an issue for empirical investigation. However, two lessons from the 30 years of computer-task enrichment are relevant to this point.

First, we have repeatedly observed that apparent manipulations of environmental enrichment may or may not have observed impact on the psychological wellbeing of the monkeys. What I am saying here is more than, "Some manipulations are effective for promoting psychological wellbeing and others are not" which is true but trivial. Moreover, some manipulations improve the appearance of environmental enrichment, whether or not they effectively change the animals' behavior and wellbeing. Conversely, other manipulations do not impact the perception of environmental enrichment, but may be highly impactful with respect to wellbeing itself. A few examples will help to illustrate this point.

Across the years, we have of course tried many ways to provide an environment that promotes the psychological wellbeing of our monkeys and apes. Undergraduate students completing practicum or honors thesis work have proposed various enrichment options, including music, movies, mirrors, and a wide range of puzzles. The monkeys ignored many of these, and some of the others enjoyed the brief attention that novelty can elicit and then faded quickly into disuse. In contrast, it is noteworthy that rhesus monkeys continue to engage the computer-task apparatus day after day, year after year. Of course, different tasks provide new challenges and stimulation, but we do not see declines across time in the number of trials completed, the amount of time spent in task-related activity, or the effectiveness of the computer-test paradigm in reducing symptoms of boredom or psychological distress. (However, it must be acknowledged that occasions in which the computer-task paradigm malfunctions - a joystick stops working correctly, the pellet dispenser jams - appear quite frustrating and stressful for the monkeys, who not only lose the benefits of task-directed activity but who also experience a similar frustration that might be similar to what we human feel when our technology fails. Fortunately for the monkeys, these instances are rare and shortlived.)

Each year, the LRC is contacted by numerous individuals and groups to inquire about tours. Unfortunately, we are unable to accommodate most of these requests, as such visits are highly disruptive to testing. Occasionally, select groups are approved for visits however. Answers were obtained from several such tour groups to the question, "Which of the following would be effective in making the monkeys happier?" followed by the list of potential interventions in Table 1. The table summarizes how visitors thought about each potential intervention, and also what our experience indicates about the effects of each intervention on wellbeing index. 
Table 1

Perceived and Observed Enrichment Value of various Options

\begin{tabular}{ccc}
\hline Enrichment Option & Perceived Impact & Observed Impact \\
\hline Cage-size increase & High & Moderate or low \\
Climbing/swinging structures & High & High for young monkeys \\
Computer games & High & High \\
Conspecifics in same room & Moderate & High \\
Foraging toys (e.g., Kong ball) & Low & High when baited, low after \\
Infant toys (e.g., rattles) & Moderate & High when novel, low after \\
Menu options & Low & Moderate to high \\
Mirrors & Low & Low for most monkeys \\
Music/radio & High & Low \\
Musical/noisy toys & High & Low (with some neophobia) \\
Outdoor access & High & High \\
Pair housing & High & Generally high if compatible \\
Pictures/painting on walls & High & Low \\
Playset (e.g., toddler slide) & High & Low \\
Television or Movies & High & Low for most monkeys
\end{tabular}

The obvious interpretation of these data is that there is little correlation between visitors' perception and observed impact of enrichment interventions. This is not shocking, given that most of these visitors are not experts on primate behavior. However, the results are meaningful for reasons beyond this conclusion. When people see or even think about laboratory research with nonhuman primates (or primates on display at a zoo for that matter), there is considerable anthropomorphism in their evaluations of the animals' environment and wellbeing. Novak and Suomi (1988) discussed the risks of this type of anthropomorphism, and championed the necessity of empirical assessment of putative enrichment options. It remains clear that the "an environment adequate to promote the psychological wellbeing of nonhuman primates" should be measured against the animals' standards, not assumptions based on human happiness. On the other hand, the anthropomorphic perception of an enriched environment is not unimportant, even if it fails to map directly onto the animals' experience of enrichment. With the exception of some music- or noise-producing toys that we tried (e.g., electronic devices made for children that generate sounds when buttons are pressed), which frequently appeared to frighten some of our monkeys, there may be no harm in manipulations that look enriching even if they do not really benefit the monkeys. There is value in inexpensive interventions that look like they might make the monkeys happier - that people think, "I would like that if I were a monkey"-so long as those interventions do not actually make the monkey less psychologically well.

Unfortunately, naturalistic manipulations (tree branches, natural substrate, and so forth) were not included in this study. There seems little doubt that making the laboratory look like nature will impact the perception of how enriching the environment is, and again I believe that there is great value in that perception. It remains for empirical tests to determine whether such changes are also uniformly effective in actually improving an animal's psychological wellbeing.

The best options would seem to be those interventions that both yield the perception of being enriching and also improve psychological wellbeing effectively. Visitors rated "computer games" as highly enriching, and although the simple graphics and demands of our cognitive tasks pale in comparison to most commercial computer games even for very young children, we have seen that these game-like tasks are indeed effective in promoting psychological wellbeing. Nevertheless, I suspect that there are experts on primate behavior who remain skeptical about computer-based enrichment, noting accurately that it generates activity that is quite unlike the naturalistic behavior of nonhuman primates in the field. One does not see computer-using monkeys and apes outside the laboratory, although I often joke about how glorious it would be to mount joysticks and computers to trees in the wild. Notwithstanding, I wish to make the case that there is nothing unnatural about rhesus monkeys and other nonhuman primates completing computerbased tasks. Looking beyond the obvious differences, these computer tasks tap many of the same adaptations and require many of the same behaviors as are required for survival in nature. Nonhuman primates are flexible. Faced with new challenges, they learn, adapt, and thrive. Whether rhesus monkeys 
are foraging for food in nature by looking for seeds and insects on the ground, or they are foraging for food in the lab by manipulating a joystick so as to earn pellets of fruit-flavored chow, the quest for nutrition and the capacity to learn how to get it is constant. What I am arguing is that there is almost nothing more natural for a rhesus monkey than matching-to-sample (MTS - although I would make the same argument for delayed matching, or two-choice discrimination learning, or any other cognitive task that is within the animals' competencies). From the dawn of time, and certainly from birth, everything with the monkeys' genetic and experiential history has prepared them for the challenges of MTS. This claim is not based on a teleological story about how survival and reproduction in the wild depends on MTS, as in candor there may be no activity in the wild that quite resembles the demands of a MTS task. However, the nonhuman primate faces many moments in nature when it must learn "What do I need to do in this situation to obtain food (or safety, or a reproductive partner, and so forth)?" Rhesus monkeys and other nonhuman primates must learn from experience, transfer learning to positive advantage in new contexts, and solve problems. The problems may not look like MTS, but this computerized task is just one example of the kinds of problems that primates are good at solving. It is this adaptive capacity to learn and to thrive in the face of new challenges that makes rhesus monkeys - and other primates, including humans - such hearty, resourceful, and resilient animals. It is this same capacity that is required every day in nature. It is this same capacity that is required every day they use the computer-test system in the laboratory. Manipulating a joystick or responding to a touchscreen might not look exactly like foraging in the wild, but the difference is technological, not psychological.

Many primate experts have appropriately cited the importance of natural behaviors in an animal's repertoire as an indicator of psychological wellbeing. As discussed above, this was one of the four pillars of the wellbeing definition advanced by Novak and Suomi (1988; see also Markowitz and Spinelli, 1986). In part, this reflects earlier findings of deficits in behavioral (e.g., Harlow \& Suomi, 1970) and cognitive (e.g., Davenport, Rogers, \& Rumbaugh, 1973) competence for animals raised in impoverished environments. It is important to note however that the importance of a behavioral repertoire that is typical of animals in the wild does not mean that psychologically well animals only behave in ways that are observed in free-ranging counterparts. Rather, they should show the range of competencies that would be expected of the species outside of the lab.

For obvious reasons-including those related to the animals' psychological and physical wellbeing - captive primates generally do not experience the threats of predation, disease, starvation, or environmental disasters. But these are mere conditions that evoke naturalistic behaviors, as are the characteristics of food availability, travel range, social and reproductive opportunity, and so forth. Environmental enrichment does not need to replicate the conditions of nature to be effective; however, to impact psychological wellbeing positively, an enrichment intervention does need to address one or more of the basic, natural needs of nonhuman primates.

\section{Lesson 4: The Four Cs of Psychological Wellbeing}

Considering the extensive literature that has been produced in the aftermath of the 1985 amendment of the AWA, as well as our own experience with the resident monkeys and chimpanzees at the LRC, I am convinced that the psychological wellbeing of captive primates reflects four basic factors or needs: comfort, companionship, challenge, and control. Efforts to provide effective environmental enrichment must tap one or more of these four dimensions of psychological wellbeing. Each will be defined and discussed briefly below, as will illustrative evidence of whether/how the computerized test system relates to these "four Cs."

Comfort. An animal's comfort needs include all variables associated with the animal's physical health and freedom from pain, discomfort, or environmental stressors. Animals that are physically ill or experiencing pain will have compromised psychological wellbeing. Animals that are healthy but housed in conditions that are too hot, too cold, too small, or otherwise uncomfortable will have compromised psychological wellbeing. Animals that experience chronic threat or are otherwise under survival stress will have compromised psychological wellbeing. 
The computerized test system is an enrichment option that is consistent with the physical health and comfort of the animals, although at the LRC we have looked for ways to encourage the animals to move around the cage more, so they do not become game-playing couch potatoes. For example, putting the joystick and pellet cup at the top of the home cage, but the water lixit at the bottom of the cage, forces the monkey to climb up and down frequently for exercise. It also provides a method for the monkeys to feed themselves, and it is possible to control the distribution and type of food in this way. However, the computerized test system is basically value-neutral with respect to a monkey's physical health and comfort.

This is not to suggest that the Rumbaughx paradigm has no valuable with respect to animal health. Changes in activity and diet - including computer-task activity and the motivation to earn and to consume nutritive rewards - are key indicators of physical health. Across the years at the LRC, monkeys have occasionally gotten ill or suffered physical problems (e.g., digestive blockage, tooth problems). Early symptoms of this malaise included atypical listlessness, accompanied by precipitous declines in taskdirected activity. Such inactivity stands in sharp contrast to the monkeys' typical levels of task engagement, and elicits attention from researchers, caregivers, and veterinarians. Even subclinical variations in physical wellness might result in detectable alterations in task productivity and performance, as in the unpublished feasibility study in which our monkeys consumed juice with low dosages of alcohol or Diazepam, which produced significant performance deficits despite a complete absence of overt behavioral manifestations of drug consumption.

Recall the Rhesus Project discussed earlier. The international, interdisciplinary project team finally realized the 14-day spaceflight of rhesus monkeys in 1986. Two joystick-trained monkeys spent 14 days orbiting the Earth, while two control animals experienced matched conditions with the exception of the spaceflight. Whereas no changes were observed in computer-task performance for control animals, computer-task latency was significantly longer after 14 days in microgravity (Washburn et al., 2000). Again, this suggests that computer-task performance may be sensitive to variations in physical health and related variables subsumed under the Comfort factor of psychological wellbeing.

Companionship. Probably no variable related to the psychological wellbeing of captive primates has been discussed more than the social-housing variable. With highly social apes and capuchin monkeys also at the LRC, it is easy to convince oneself that companionship is unimportant to our colony of adult male rhesus monkeys (and their well-earned reputation for being socially difficult!) — until, as we unfortunately experienced recently, an old monkey dies and we see the decline in psychological wellbeing of its long-time roommate.

Evidence for the potency of social-housing manipulations on psychological wellbeing was discussed above (Figure 2). I will note, however, that Comfort needs appear to be more fundamental than Companionship needs in a primate's psychological wellbeing. Pair-housing can be wonderful for a dominant animal but highly stressful or even physically risky for the subordinate. Psychological wellbeing indices may decline under conditions of social housing if space is inadequate, if competition for resources is created, or if conflict emerges. Procedures for introducing animals into pair- or group-housing have been studied and described, but even the most careful procedures cannot completely eliminate the risk of serious injuries or high levels of stress. Animals that have lived together peacefully for many years may suddenly fight violently, perhaps in response to some external stressor, but perhaps inexplicably and unpredictably. In such instances, the animal's health, safety and comfort needs trump the companionship needs, and the latter may be addressed, albeit incompletely, with visual and auditory access of animals that are safely separated.

The computerized test system is certainly compatible with the companionship needs of nonhuman primates. Washburn and Rumbaugh (1991) reported that task performance declined significantly when animals were socially isolated compared to when they were tested together. Gazes and colleagues (2013) reported that macaques produced similar levels of performance when they were tested in a group or individually (i.e., in separate cages within the same room). The group testing on computerized tasks in this study used the radio-frequency identification system described by Fagot and Bonté (2010), in which tiny transmitters inserted subcutaneously in each monkey's arm identifies the animal as it reaches to respond to a trial (e.g., to use a touchscreen), which in turn automatically administers the appropriate task and condition 
for the specific animal, and records the monkey's identity with the data. This system makes it possible efficiently to train and to test groups of nonhuman primates, without separating them from conspecifics for testing. Without doubt, it is an innovation that will promote psychological wellbeing by combining the benefits of the computer-task paradigm while addressing the Companionship needs of the participant animals.

Challenge. Nonhuman primates require stimulation. They need puzzles to solve, things to learn, challenges to overcome. They are curious. The computerized test system is unsurpassed as an instrument for capitalizing on these attributes, and for providing a great variety of challenges that can be tailored to the individual skill levels, experience, and interests of the animals. In research at the LRC, rhesus monkeys engage in the tasks even when other enjoyable activities are available. They spend considerable time engaged in task-directed behavior, even though they receive enough food daily whether or not they work. Indeed, in tests in which chow is continuously available in abundance, the animals will continue to engage the computer tasks, albeit producing significantly fewer trials per day (in part because they are napping much more often!), even if they choose not to consume the pellet rewards. If one disconnects the pellet dispensers, the animals will become frustrated and stop working - as they do if the dispensers jam or malfunction; however, if one leaves the dispensers disconnected, the animals will eventually re-engage the tasks and work in the absence of nutritive rewards. Again, they do not perform thousands of trials per day in this situation, as is typical when the animals receive pellets for correct responses, but they do clearly work on the tasks even in the absence of food reward. The pellet rewards are certainly an important part of the motivation to engage the tasks, but the evidence suggests that the animals are also motivated by enjoyment of the tasks themselves. Indeed, given a choice between completing trials for pellets or receiving pellets for free but not being able to play the game-like tasks during the free-pellet period, the monkeys choose to work for their reward (Washburn \& Rumbaugh, 1992a). Even free pellets are too costly if they mean that the monkeys will lose access to the computer-test system for five minutes!

The motivational value of the computer-test system is also illustrated by the finding that monkeys choose to work on tasks of various levels of difficulty (Washburn et al., 1991). Although the monkeys' most preferred tasks tend to be those richest in reward density, the animals also choose to work on more difficult and less rewarding tasks. Preference for a variety of tasks and challenges, rather than always selecting the easiest task that produces the most pellets/minute shows that rhesus monkeys may be greedy, but they are not lazy. The Challenge dimension of their psychological wellbeing demands variety, stimulation, and perhaps even the accomplishment associated with succeeding on difficult tests.

Control. The least extensively studied component of psychological wellbeing of nonhuman primates is choice, or control. To the degree possible, a nonhuman primate's environment should afford options or choices - over the what, where, when, with whom, and why (for what) of activities. A guiding principle of testing at the LRC since it was founded by Duane Rumbaugh is that nonhuman primates should, inasmuch as is possible, volunteer to contribute to research activities. The rhesus monkeys work or rest ad libitum. They choose what tasks to engage, either from a menu of icons or simply by declining to work on tasks in which they are not interested. In this way, we believe that we get data at those times that the monkeys are most highly motivated to work, and to work specifically on the task that they have chosen to engage. We have also reported that task performance (trials produced, response accuracy, response latency) are improved when the animals are given choice over the tasks compared to when those same tasks are administered in a yoked order, but without choice (Washburn \& Rumbaugh, 1991). Recently, Perdue and collaborators (2014) showed that rhesus monkeys will choose to choose. That is, given the choice between choosing their tasks from a menu or having the tasks assigned to them, the monkeys prefer to have choice and control.

The importance of choice and control for psychological wellbeing brings to mind a related lesson from these decades of computer-task research with rhesus monkeys: Where possible, it seems preferable to allow the animals themselves to indicate the conditions that are enriching. That is, if one takes seriously the assumption that nonhuman animals want to be psychologically well, then the animals' own choices should be the ultimate standard for what environmental manipulations are truly enriching. Rather than making assumptions about the conditions that will promote psychological wellbeing, we should endeavor to give 
the animals choices that will indicate those conditions. For example, rather than assuming that social access to conspecifics is always preferable to single housing, we have begun to teach the rhesus monkeys icons or symbols that will allow them to request pair housing (or outdoor access, or particular foods or treats, and so forth). We propose that allowing animals to indicate their preferences by their behavior provides the clearest and most defensible standard for determining environmental enrichment, and in any case that the opportunity to make the choice contributes to the Control component of psychological wellbeing.

Summary. Although these four Cs of psychological wellbeing are suggested by experience at the LRC with monkeys and apes, they also seem to be consistent with the broader literature on the psychological wellbeing of nonhuman primates, which includes many other species. Indeed, I believe that these same four dimensions are important for the psychological wellbeing of human primates. I reviewed the literature on the psychological wellbeing of aged adults who reside in assisted living or hospital settings. Such studies reveal a number of considerations or factors that determine happiness of such individuals, but all of these fit within the categories of health/comfort (including mobility), companionship, challenge, and control.

In conclusion, the 1985 amendment to the Animal Welfare Act served to stimulate a tremendous amount of excellent research and scholarly debate. Four of the lessons this author has learned from 30 years of research partnership with nonhuman primates are discussed here, largely stemming from extensive use of a particular computer-based strategy for environmental enrichment. As innovative and effective as the computer-task paradigm has been, it certainly has never carried the primary burden of ensuring the psychological wellbeing of the monkeys and apes at the LRC. That responsibility has been expertly borne by a host of talented and dedicated faculty/staff/student researchers, caretakers, and veterinarians who recognize the privilege of stewardship that we have for these extraordinary nonhuman primates. With continued attention to the comfort, companionship, challenge and control needs of these resident animals, we hope to continue for many years to learn about the similarities and differences across primate species (including humans) in the manifestations of social and cognitive competencies, including those related to psychological wellbeing.

\section{Acknowledgments}

The research summarized here was supported by the National Institute of Child Health and Human Development (HD060563 and HD38051), by the National Aeronautics and Space Administration (NAG2438), and by Georgia State University. Across the years, this work has benefitted from the effort of more graduate and undergraduate student collaborators and staff caretakers than can be acknowledged by

name. Many Language Research Center scientists have also collaborated on these studies, and Duane Rumbaugh's contributions to almost all of this work merits particular gratitude.

\section{References}

Andrews, M. W. (1993). Video-task paradigm extended to Saimiri. Perceptual and Motor Skills, 76, $183-191$. doi:10.2466/pms.1993.76.1.183

Bayne, K. (1989). Resolving issues of psychological wellbeing and management of laboratory nonhuman primates. In E. F. Segal (Ed.), Housing, care and psychological wellbeing of captive and laboratory primates (pp. 2739). Park Ridge, NJ: Noyes Publications.

Beran, M. J., \& Minahan, M. F. (2000). Monitoring spatial transpositions by bonobos (Pan paniscus) and chimpanzees (P. troglodytes). International Journal of Comparative Psychology, 13, 1- 15.

Brannon, E. M., Andrews, M. W., \& Rosenblum, L. A. (2004). Effectiveness of video of conspecifics as a reward ror socially housed bonnet macaques (Macaca radiata). Perceptual and Motor Skills, 98, 849- 858. doi:10.2466/PMS.98.3.849-858

Clarke, A. S., Juno, C. J., \& Maple, T. L. (1982). Behavioral effects of a change in the physical environment: A pilot study of captive chimpanzees. Zoo Biology, 1, 371-380. doi:10.1002/zoo.1430010411

Clay, A. W., Perdue, B. M., Gaalema, D. E., Dolins, F. L., \& Bloomsmith, M. A. (2011). The use of technology to enhance zoological parks. Zoo Biology, 30, 487-497. doi:10.1002/zoo.20353 
Davenport, R. K., Rogers, C. M., \& Rumbaugh, D. M. (1973). Longterm cognitive deficits in chimpanzees associated with early impoverished rearing. Developmental Psychology, 9, 343-347. doi:10.1037/h0034877

Evans, T. A., Beran, M. J., Chan, B., Klein, E. D., \& Menzel, C. R. (2008). An efficient computerized testing method for the capuchin monkey (Cebus apella): Adaptation of the LRC-CTS to a socially housed nonhuman primate species. Behavior Research Methods, 40, 590-596. doi:10.3758/BRM.40.2.590

Fagot, J., \& Bonté, E. (2010). Automated testing of cognitive performance in monkeys: Use of a battery of computerized test systems by a troop of semi-free-ranging baboons (Papio papio). Behavior Research Methods, 42, 507- 516. doi:10.3758/BRM.42.2.507

Fagot, J., Gullstrand, J., Kemp, C., Defilles, C., \& Mekaouche, M. (2014). Effects of freely accessible computerized test systems on the spontaneous behaviors and stress level of Guinea baboons (Papio papio). American Journal of Primatology, 76, 56- 64. doi:10.1002/ajp.22193

Fagot, J., \& Paleressompoulle, D. (2009). Automatic testing of cognitive performance in baboons maintained in social groups. Behavior Research Methods, 41, 396-404. doi:10.3758/BRM.41.2.396

Fragaszy, D. M., Kennedy, E., Murnane, A., Menzel, C., Brewer, G., Johnson-Pynn, J., \& Hopkins, W. (2009). Navigating two-dimensional mazes: Chimpanzees (Pan troglodytes) and capuchins (Cebus apella sp.) profit from experience differently. Animal Cognition, 12, 491-504. doi:10.1007/s10071-008-0210-Z

Gazes, R. P., Brown, E. K., Basile, B. M., \& Hampton, R. R. (2013). Automated cognitive testing of monkeys in social groups yields results comparable to individual laboratory-based testing. Animal Cognition, 16, 445- 458. doi:10.1007/s10071-012-0585-8

Gulledge, J. P., Rumbaugh, D. M., Fernandez-Carriba, S., \& Washburn, D. A. (2015). Judgments of monkeys' (Macaca mulatta) facial expressions by humans: Does housing condition "affect" countenance? Psychological Record, 65, 203-207.

Harlow, H. F., \& Suomi, S. J. (1970). Nature of love-simplified. In C. M. Harlow (Ed.), From learning to love: The selected papers of H. F. Harlow (pp. 121-134; 295-304). New York: Praeger.

Hopkins, W. D., Washburn, D. A., \& Hyatt, C. W. (1996). Video-task acquisition in rhesus monkeys (Macaca mulatta) and chimpanzees (Pan troglodytes): A comparative analysis. Primates, 37, 197-206. doi:10.1007/BF02381407

Inoue, S., \& Matsuzawa, T. (2009). Acquisition and memory of sequence order in young and adult chimpanzees (Pan troglodytes). Animal Cognition, 12, S59-S69. doi:10.1007/s10071-009-0274-4

Jones, S. M., Pearson, J., DeWind, N. K., Paulsen, D., Tenekedjieva, A., \& Brannon, E. M. (2014). Lemurs and macaques show similar numerical sensitivity. Animal Cognition, 17, 503-515. doi:10.1007/s10071-0130682-3

Leighty, K. A., \& Fragaszy, D. M. (2003). Joystick acquisition in tufted capuchins (Cebus paella). Animal Cognition, 6, 141-148. doi:10.1007/s10071-003-0176-9

Mallavarapu, S., Bloomsmith, M. A., Kuhar, C. W., \& Maple, T. L. (2013). Using multiple joystick systems in computerised enrichment for captive orangutans. Animal Welfare, 22, 401-409. doi:10.7120/09627286.22.3.401

Markowitz, H., \& Spinelli, J. (1986). Environmental engineering for primates. In K. Benirsehke (Ed.), Primates: The road to self-sustaining populations (pp. 489-498). New York: Springer-Verlag.

Menzel, E. J., \& Menzel, C. R. (2007). Do primates plan routes? Simple detour problems reconsidered. In D. A. Washburn (Ed.), Primate perspectives on behavior and cognition (pp. 175-206). Washington, DC: American Psychological Association. doi:10.1037/11484-014

Novak, M. A., \& Suomi, S. J. (1988). Psychological wellbeing of primates in captivity. American Psychologist, 43, 765-773. doi:10.1037/0003066X.43.10.765

Ogden, J. J., Lindburg, D. G., \& Maple, T. L. (1993). Preference for structural environmental features in captive lowland gorillas (Gorilla gorilla gorilla). Zoo Biology, 12, 381-395. doi:10.1002/zoo.1430120408

Perdue, B. M., Clay, A. W., Gaalema, D. E., Maple, T. L., \& Stoinski, T. S. (2012). Technology at the zoo: The influence of a touchscreen computer on orangutans and zoo visitors. Zoo Biology, 31, 27-39. doi:10.1002/zoo.20378

Platt, D. M., \& Novak, M. A. (1997). Videostimulation as enrichment for captive rhesus monkeys (Macaca mulatta). Applied Animal Behaviour Science, 52, 139-155. doi:10.1016/S0168-1591(96)01093-3

Perdue, B., Beran, M. J., Rumbaugh, D. M., \& Washburn, D. A. (2014). Do monkeys choose to choose? Learning \& Behavior, 42, 164-175. 
Rumbaugh, D. M., Richardson, W. K., Washburn, D. A., Savage-Rumbaugh, E. S., \& Hopkins, W. D. (1989). Rhesus monkeys (Macaca mulatta), video tasks, and implications for stimulus-response spatial contiguity. Journal of Comparative Psychology, 103, 32-38.

Spinelli, S., Pennanen, L., Dettling, A. C., Feldon, J., Higgins, G. A., \& Pryce, C. R. (2004). Performance of the marmoset monkey on computerized tasks of attention and working memory. Cognitive Brain Research, 19, 123-137. doi:10.1016/j.cogbrainres.2003.11.007

Tanaka, M., \& Uchikoshi, M. (2010). Visual preference in a human-reared agile gibbon (Hylobates agilis). Primates, 51, 63-67. doi:10.1007/s10329-009-0175-9

Torou, L. R., Kuhar, C. W., Adcock, D., Bloomsmith, M. A., \& Maple, T. L. (2004). Computer-assisted enrichment for zoo-housed orangutans (Pongo pygmaeus). Animal Welfare, 13, 445-453.

Vonk, J., Torgerson-White, L., McGuire, M., Thueme, M., Thomas, J., \& Beran, M. J. (2014). Quantity estimation and comparison in western lowland gorillas (Gorilla gorilla gorilla). Animal Cognition, 17, 755-765. doi:10.1007/s10071-013-0707-y

Washburn, D. A. (2003). The games psychologists play (and the data they provide). Behavior Research Methods, Instruments, and Computers 35, 185-193.

Washburn, D. A., Beran, M. J., Evans, T. A., Hoffman, M. L., \& Flemming, T. M. (2013). Technological innovations in comparative psychology: From the problem box to the 'Rumbaughx'. In L. Labate (Ed.), Handbook of technology in psychology and psychiatry.179-205, Hauppauge, NY: Nova Science Publishers.

Washburn, D. A., Harper, S., \& Rumbaugh, D. M. (1994). Computer-task testing in the social milieu. Primates, 35, 343-351.

Washburn, D. A., Hopkins, W. D., \& Rumbaugh, D. M. (1991). Perceived control in rhesus monkeys (Macaca mulatta): Enhanced video-task performance. Journal of Experimental Psychology: Animal Behavior Processes, 17, 123-127.

Washburn, D. A., \& Rumbaugh, D. M. (1991). Impaired performance from brief social isolation of rhesus monkeys: A multiple video-task assessment. Journal of Comparative Psychology, 105, 145-151.

Washburn, D. A., \& Rumbaugh, D. M. (1992a). Investigations of rhesus monkey video-task performance: Evidence for enrichment. Contemporary Topics in Laboratory Animal Science, 31, 6-10.

Washburn, D. A., \& Rumbaugh, D. M. (1992b). Testing primates with joystick-based automated apparatus: Lessons from the Language Research Center's Computerized Test System. Behavior Research Methods, Instruments \& Computers, 24, 157-164. doi:10.3758/BF03203490

Washburn, D. A., Rumbaugh, D. M., \& Richardson, W. K. (1992). The Language Research Center's Computerized Test System (LRC-CTS) for environmental enrichment and psychological assessment. Contemporary Topics in Laboratory Animal Science, 31, 11-15.

Washburn, D. A., Rumbaugh, D. M., Richardson, W. K., Gulledge, J. P., Shlyk, G. G., \& Vasilieva, O. N. (2000). PTS performance by flight- and control-group macaques. Journal of Gravitational Physiology, 7, S89-S94.

Yamanashi, Y., \& Hayashi, M. (2011). Assessing the effects of cognitive experiments on the welfare of captive chimpanzees (Pan troglodytes) by direct comparison of activity budget between wild and captive chimpanzees. American Journal of Primatology, 73, 1231-1238. doi:10.1002/ajp.20995 